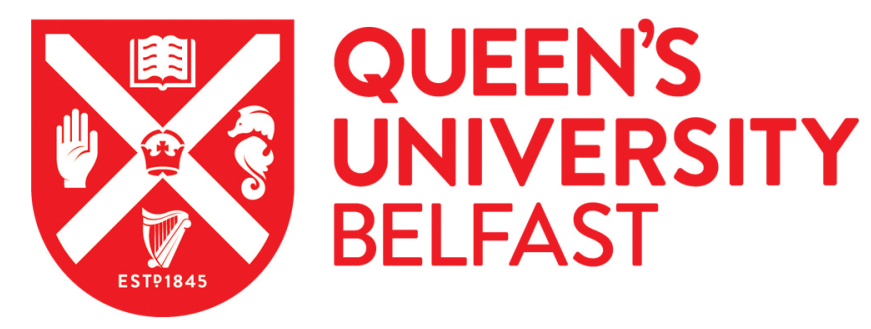

\title{
Independent particle descriptions of tunneling using the many-body quantum transport approach
}

Fagas, G., Delaney, P., \& Greer, J. C. (2006). Independent particle descriptions of tunneling using the manybody quantum transport approach. Physical Review B (Condensed Matter), 73(24), 241314-1-241314-4.

[241314]. https://doi.org/10.1103/PhysRevB.73.241314

Published in:

Physical Review B (Condensed Matter)

Queen's University Belfast - Research Portal:

Link to publication record in Queen's University Belfast Research Portal

\section{General rights}

Copyright for the publications made accessible via the Queen's University Belfast Research Portal is retained by the author(s) and / or other copyright owners and it is a condition of accessing these publications that users recognise and abide by the legal requirements associated with these rights.

Take down policy

The Research Portal is Queen's institutional repository that provides access to Queen's research output. Every effort has been made to ensure that content in the Research Portal does not infringe any person's rights, or applicable UK laws. If you discover content in the Research Portal that you believe breaches copyright or violates any law, please contact openaccess@qub.ac.uk. 


\title{
Independent particle descriptions of tunneling using the many-body quantum transport approach
}

\author{
Giorgos Fagas \\ Tyndall National Institute, Lee Maltings, Prospect Row, Cork, Ireland \\ Paul Delaney \\ School of Mathematics and Physics, Queen's University Belfast, Belfast BT7 1NN, Northern Ireland \\ James C. Greer \\ Tyndall National Institute, Lee Maltings, Prospect Row, Cork, Ireland
}

(Received 24 May 2006; published 26 June 2006)

\begin{abstract}
Currents across thin insulators are commonly taken as single electrons moving across classically forbidden regions; this independent particle picture is well-known to describe most tunneling phenomena. Examining quantum transport from a different perspective, i.e., by explicit treatment of electron-electron interactions, we evaluate different single particle approximations with specific application to tunneling in metal-molecule-metal junctions. We find maximizing the overlap of a Slater determinant composed of single-particle states to the many-body current-carrying state is more important than energy minimization for defining single-particle approximations in a system with open boundary conditions. Thus the most suitable single particle effective potential is not one commonly in use by electronic structure methods, such as the Hartree-Fock or Kohn-Sham approximations.
\end{abstract}

DOI: 10.1103/PhysRevB.73.241314

PACS number(s): 73.40.Rw, 73.63.-b, 05.60.Gg, 31.25.-v

Describing quantum transport from first principles has proven to be a challenging task; debate continues as to the proper theoretical approach for treating electron currents across metal-molecule-metal junctions (MMJs). ${ }^{1-7}$ Tunneling is an archetype of quantum behavior with implications for all branches of modern physics, as well as chemical and biological processes dominated by electron transfer. The ability to accurately describe tunneling currents on the nanoscale is important from both fundamental science and technology perspectives. The discussion focuses on the suitability of various Kohn-Sham density functional theory (KS DFT) implementations, ${ }^{1-4}$ or possibly the Hartree-Fock (HF) method, 2,6 to apply in combination with the one-body nonequilibrium Green's function (NEGF) approach. ${ }^{4,8}$ In fact though, there is no established criterion for selecting a single-particle Hamiltonian to be used in transport calculations.

On the other hand, evidence has been mounting that electron transport can be sensitive to many-body exchange and correlation. $^{2,6,7}$ For example, applying the NEGF together with static KS DFT leads to overestimation of the current in MMJs, in some cases, up to orders of magnitude when compared to experiments. ${ }^{4}$ Several reasons for discrepancies have been suggested: DFT's underestimation of the occupied-unoccupied state separation results in too high currents, ${ }^{3,9}$ conversely the overestimation of the gap in the $\mathrm{HF}$ approximation yields currents too $\mathrm{low}^{2}$; DFT exchangecorrelation functionals do not accurately reflect potential profiles ${ }^{10}$; other methods such as time-dependent current (DFT Ref. 11) or density matrix functional theory are simply better suited for the treatment of electronic currents. Irrespective of the proposals to explain or remedy single-particle tunneling descriptions, it is necessary to understand the physics deriving from a genuine many-body formalism to identify the source of the discrepancies.

In this Rapid Communication we investigate the extent of correlations beyond the single-particle picture and identify conditions for defining a "best" independent-particle model, using a recently formulated many-body quantum transport approach. ${ }^{5}$ We demonstrate a single-electron description of tunneling deriving from maximizing the overlap of a single Slater determinant with the true many-body current-carrying state, and show that it remains a good approximation even after conventional independent-particle models fail.

Most theoretical studies of quantum transport begin with the use of a Slater determinant of single-particle states to model a many-body current-carrying wave function. ${ }^{12}$ Our recent work ${ }^{5}$ finds that this picture becomes markedly less valid near or above a resonance. Here, we concentrate on nonresonant tunnel models. Independent-particle models are appealing because as the wave function can be represented as a single Slater determinant, the resulting physical model can readily be pictured and computed. ${ }^{4,13}$ Their limitation is neglect of electron correlations; below we introduce a correlation measure to quantify when an independent-particle model holds.

For our study, MMJs are an ideal test case as the molecule acts like an insulator when there is little charge transfer or hybridization with the electrodes, there is a large set of experimental observations to validate the calculations, and MMJs may be modeled with a relatively small number of atoms, allowing their electronic structure to be accurately calculated with many-body methods. We choose alkane chains $\left(\mathrm{C}_{2} \mathrm{H}_{4}\right)_{n}$ (inset of Fig. 1) and for comparative purposes also examine silicon hydride molecules $\left(\mathrm{Si}_{2} \mathrm{H}_{4}\right)_{n}$ ( $\mathrm{si}$ lanes), as replacing the carbon atoms with silicon reduces the occupied-unoccupied state separation and increases the degree of correlation.

Details of the transport method are given in Ref. 5. A constraint Ansatz using the Wigner function is made to incorporate open-system boundary conditions for calculation 


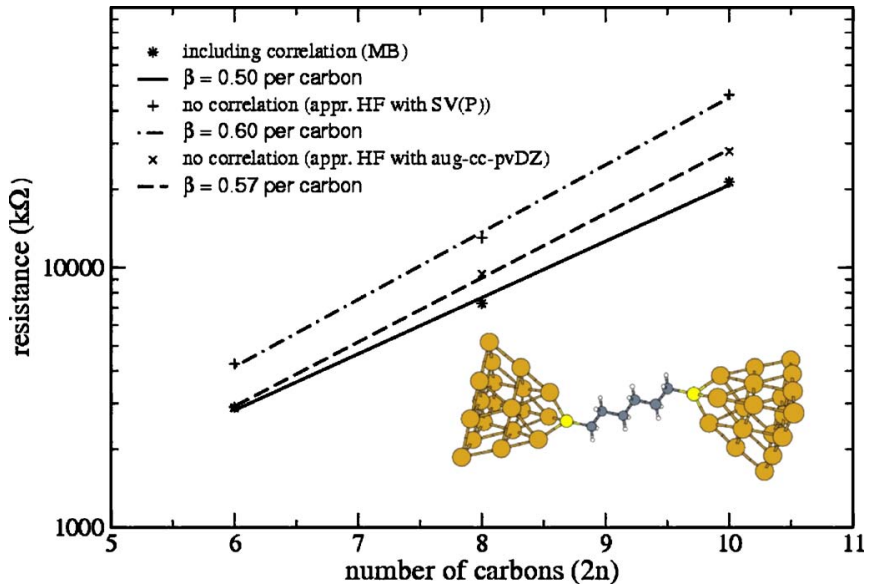

FIG. 1. (Color online) Tunnel resistance-increase exponential law. Explanation of the standard basis set notations is given in the text. Inset: Typical geometry of the studied $\mathrm{Au}_{\text {tip }}-\mathrm{S}-\left(\mathrm{C}_{2} \mathrm{H}_{4}\right)_{n}-\mathrm{S}-\mathrm{Au}_{\text {tip }}$ molecular junctions $(n=3)$.

of the reduced density matrix on a device region (typically the molecule plus part of the electrodes) at several values of applied voltage. The procedure results in the best approximation to the density matrix on a region subject to reproducing known system observables in accord with the principle of maximum entropy at the zero-temperature limit. The density matrix is calculated from eigenfunctions of a constrained many-electron Hamiltonian. A strength of our approach is that it allows for the expansion of many-body states in terms of a complete set of configurations

$$
|\Psi\rangle=c_{0}\left|\Psi_{0}\right\rangle+\sum_{i, a} c_{i}^{a}\left|\Psi_{i}^{a}\right\rangle+\sum_{i<j} \sum_{a<b} c_{i j}^{a b}\left|\Psi_{i j}^{a b}\right\rangle+\cdots
$$

$\left|\Psi_{0}\right\rangle$ refers to a reference state composed of the $N$ lowest single-particle states, $\left|\Psi_{i}^{a}\right\rangle \quad\left(\left|\Psi_{i j}^{a b}\right\rangle, \ldots\right)$ denotes singly (doubly,...) excited configurations generated by substituting the $i$ th $(j$ th,$\ldots)$ occupied single-particle state with the $a$ th $(b$ th,...) single-particle excitation. Indexed $|\Psi\rangle$ are spinprojected Slater determinants or configuration state functions (CSFs), and $\left|\Psi_{0}\right\rangle$ is the ground-state HF determinant in the absence of an applied electric field. The symmetry group of our contact-molecule-contact subsystem is $C_{2 h}$ (inset of Fig. 1) whose ground-state eigenfunction at $V=0$ is a singlet with $A_{g}$ symmetry. The bias field polarizes the molecular subsystem and mixes states of singlet $B_{u}$ symmetry via dipole terms. Due to the combinatorial nature of generating possible configurations, there is a computationally prohibitive number of excited-state determinants. To calculate the many-body tunneling wave function $\left|\Psi_{\mathrm{MB}}\right\rangle$ we approximate the full expansion (1) by taking the most significant CSFs, as computed from a Monte Carlo sampling of the many-electron expansion space ${ }^{14}$ with the addition of all singly excited CSFs. Notably, this approximation is not excitation limited and allows for a high degree of electron correlation. ${ }^{15}$

In contrast to the many-body wave function, a single Slater determinant $\left|\Psi_{1 \text {-det }}\right\rangle$ is sought in independent-particle models. If this is initially built from a set of unperturbed single-particle states, e.g., the Hartree-Fock $\left|\Psi_{0}\right\rangle$ as above, and a perturbation such as an electric field is introduced, the resulting many-body expansion of the perturbed single- determinant state has the special feature that the coefficients of the doubly (triply,...) excited configurations will be appropriate products of the coefficients of the singly excited configurations

$$
\left|\Psi_{1-\text { det }}\right\rangle \approx\left|\Psi_{0}\right\rangle+\sum_{i, a} c_{i}^{a}\left|\Psi_{i}^{a}\right\rangle+\sum_{\substack{i<j \\ a \neq b}} c_{i}^{a} c_{j}^{b}\left|\Psi_{i j}^{a b}\right\rangle+\ldots
$$

That this is the most general determinant not orthogonal to $\left|\Psi_{0}\right\rangle$ is seen by expanding the Thouless expression $\left|\Psi_{1 \text {-det }}\right\rangle$ $=\exp \left(\sum_{i, a} c_{i}^{a} \hat{a}_{a}^{\dagger} \hat{a}_{i}\right)\left|\Psi_{0}\right\rangle$, where $a_{i}^{\dagger}\left(a_{i}\right)$ creates (annihilates) the $i$ th orbital. From the many-body calculation we find that there is a broad voltage range where linear response is valid; then by taking terms up to second order in the $c_{i}^{a}$ in (2) and applying the same Wigner function transport scheme to this restricted form of the wave function, we deduce the HF results to linear response in the bias. This permits comparison of the exact-exchange (but uncorrelated) and correlated descriptions of tunneling within the same formalism.

In Fig. 1 both the correlated and uncorrelated tunnel resistance of the alkane-based molecular junctions are shown. The qualitative features are the exponential suppression $R=R_{0} e^{\beta N_{\mathrm{c}}}$ of the current with respect to the number of carbon atoms $N_{\mathrm{c}}=2 n$ (Fig. 1) and linear scaling with applied voltage. As observed in numerous experiments, both results are typical of a metal-insulator-metal system. Contact resistances $R_{0}$ and tunneling parameters $\beta$ calculated by leastsquare linear fits are relatively close in value with HF $R_{0}$ and $\beta$ deviating from the many-body results by $32 \%$ and $14 \%$, respectively. The overestimation of $\beta$ by the HF approximation can be interpreted in terms of the incorrect alignment of the virtual orbitals, which leads to a larger highest occupiedlowest unoccupied molecular orbital gap. In contrast, the contact resistance $R_{0}$ is underestimated as indicated by the zero crossing in Fig. 1, suggesting a stronger moleculeelectrode coupling within the HF results. Similar behavior is found for the silane chains, with $\beta=0.18$ per silicon and a contact resistance of $R_{0}=900 \mathrm{~K} \Omega$ from the many-body calculation. As expected, these molecular junctions are much more conducting, yielding a value of $\beta \approx 0.09 \AA^{-1}$ compared to $\beta \approx 0.39 \AA^{-1}$ for alkanes. But, as will be discussed in detail, the silanes display a higher degree of electron correlation and the HF deviations with respect to the many-body results are substantially larger than for the alkanes, increasing to $75 \%$ and $117 \%$ for $R_{0}$ and $\beta$, respectively.

Quantitatively, the magnitudes of the low-bias resistance for each alkane molecule compare well with those reported in Ref. 16 apart from decane, which appears to be experimentally poorly resolved, as the measured result for $n=5$ does not fit the extrapolated tunneling behaviour. Our estimated inverse decay length $\beta$ falls in the lower part of the experimentally observed range of values scattered between about 0.5 and 1.0 per carbon atom. ${ }^{17}$ In particular, Haiss et $a l .{ }^{18}$ have reported $\beta=0.52 \pm 0.05$ per carbon but with higher resistance values (compare to values indicated in Fig. 1).

We can state that the HF theory performs relatively well compared to a full many-body calculation for nonresonant transport in the alkanes, but eventually diverges from the many-body result as the molecular length increases. Due to the higher degree of correlation, the HF is a poorer approxi- 


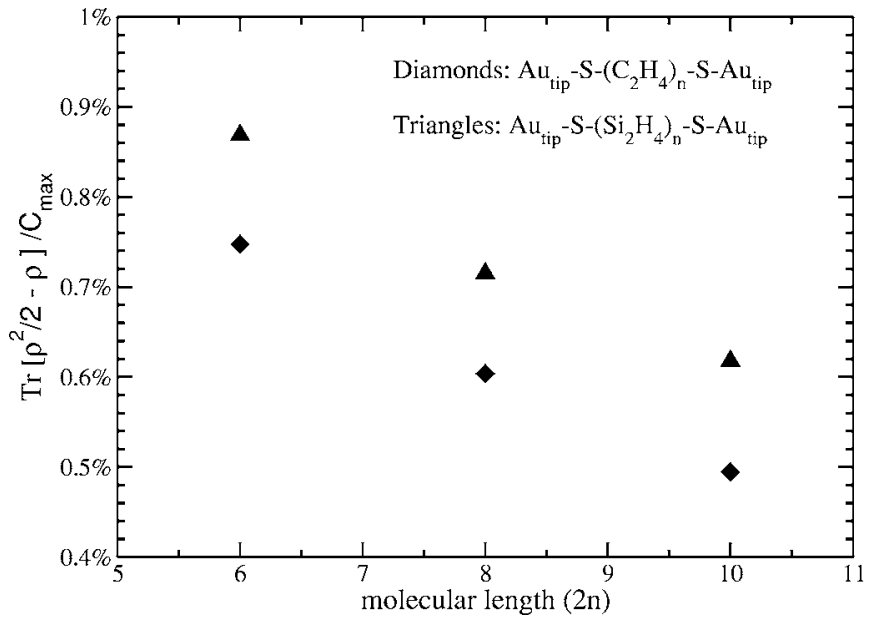

FIG. 2. Many-body correlations expressed via the one-body density matrix. These are voltage independent with a standard deviation much smaller than the symbol size.

mation for the silanes, and also diverges with chain length as compared to the many-body calculation. In Fig. 1, the HF results for the smaller but commonly used atomic orbital split valence with polarization $[\mathrm{SV}(\mathrm{P})]$ basis set for the carbon atoms is included (the larger aug-cc-pvDZ set denotes a valence double- $\zeta$ correlation-consistent basis set with polarization, augmented with diffuse functions). Within this approximation, the molecular gap becomes larger, yielding a small increase in $\beta$ and higher resistance. This result does not compare as well to the many-body calculation and casts doubts on results whose convergence has not been tested with respect to the completeness of the single-particle basis.

We have examined the importance of electron correlation by comparing separate calculations at the many-body and HF levels; however, the level of correlation may be directly quantified from the many-body calculation independently of HF results by examining the one-body density matrix $\gamma_{1}$. Lack of correlations is expressed by the condition that $\gamma_{1}$ may be derived from a single determinant if and only if it is idempotent, $\operatorname{Tr}\left[\hat{\gamma}_{1}^{2}-\hat{\gamma}_{1}\right]=0$. For closed-shell systems such as those studied here, the degree of correlation $C \geq 0$ - or equivalently the deviation from idempotency-for the spintraced density matrix $\rho_{1}$ may be defined in terms of its eigenvalues or natural occupation numbers $n_{i}$ as

$$
C=\frac{1}{N-1} \sum_{i} n_{i}\left(2-n_{i}\right) / 2 \text {. }
$$

As $0 \leq n_{i} \leq 2$, an upper bound can be deduced:

$$
C_{\max }=\frac{N}{2(N-1)}\left(2-\frac{N}{N_{T}}\right) \text {. }
$$

Here $N$ is the total number of electrons in the device region and $N_{T}$ is the size of the single-particle basis. The magnitude of $C$ is a direct measure of the deviation of the actual onebody density matrix from an uncorrelated one deriving from a determinantal wave function; $C=0$ for a single determinant and $C=C_{\max }$ when $n_{i}=N / N_{T}$.

In Fig. 2, we plot the correlation measure $C$ scaled to its upper limit as a function of chain length for $\left|\Psi_{\mathrm{MB}}\right\rangle$ (note that it vanishes for $\left.\left|\Psi_{1 \text {-det }}\right\rangle\right)$. Remarkably, as a function of voltage

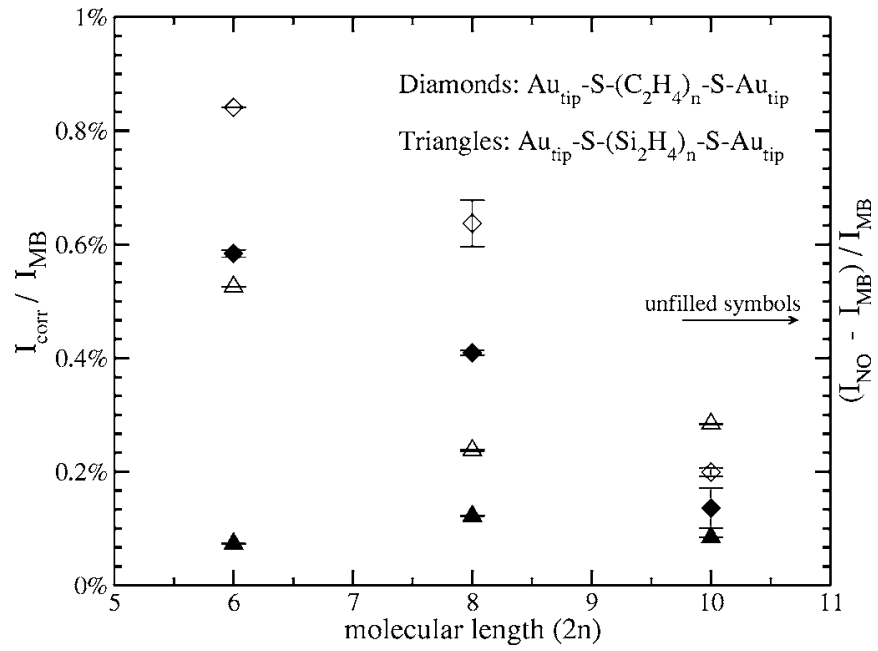

FIG. 3. (Left axis) Contribution of correlations to the current measured via Eq. (5). (Right axis) Current percentage not carried by the leading determinant defined as the $N$-particle configuration with highest natural occupancies. Residual noise voltage dependence is indicated by error bars.

(not shown) the correlation measure is constant and close to the uncorrelated limit for any given oligomer. In fact, $C$ monotonically decreases with increasing length possibly approaching a lower limit set by an infinite polymer for both the alkanes and the silanes, the latter system displaying more electronic correlation. Hence, in contrast to the HF predictions, the density matrix reveals that there exists a single Slater determinant description that is equally valid for all chain lengths.

A formal measure of correlation for any expectation value $\left\langle\Psi_{\mathrm{MB}}|\hat{I}| \Psi_{\mathrm{MB}}\right\rangle$ can be devised by using the fact that the spin-traced two-body density matrix $\rho_{2}$ factorizes in terms of $\rho_{1}$ only in the one-determinant (uncorrelated) approximation. We write $\rho_{2}(i j ; k l)=(1 / 2)\left[\rho_{1}(i k) \rho_{1}(j l)\right.$ $\left.-\rho_{1}(i l) \rho_{1}(j k) / 2\right]+\lambda(i j ; k l)$, where the first term is familiar from HF theory and $\lambda$ denotes any deviations when $\rho_{1}$ is not idempotent. Here $i$ and $k$ are indices of the first electron, $j$ and $l$ that of the second, and $\sum_{i, j} \rho_{2}(i j ; i j)=N(N-1) / 2$. Trac-

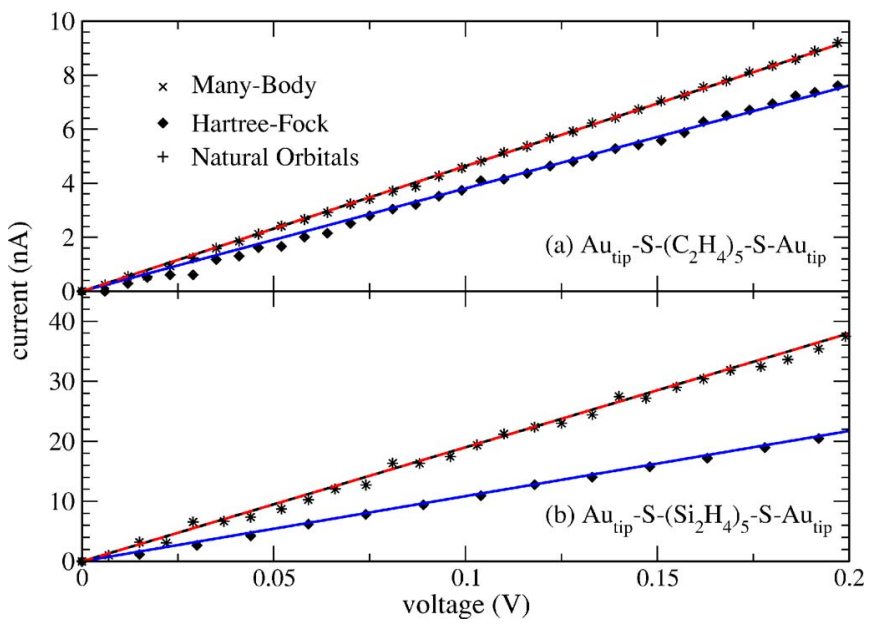

FIG. 4. (Color online) Comparison of the many-body, HartreeFock, and leading natural orbital determinant currents. 
ing over the coordinates of the second electron, we deduce a partition of $\rho_{1}=1 /(N-1)\left[N \rho_{1}-\rho_{1} \times \rho_{1} / 2\right]+\lambda$ into uncorrelated and correlated pieces. This gives us a decomposition of the current $\langle\hat{I}\rangle=\operatorname{Tr}\left[\hat{\rho}_{1} \hat{I}\right]$ flowing in the many-body wave function as $I_{\mathrm{MB}}=I_{1 \text {-det }}+I_{\text {corr }}$, where $I_{\text {corr }}$ includes the contributions from $\lambda$ and does not vanish when $\rho_{1}$ derives from a correlated wave function. It follows that

$$
I_{\text {corr }}=\frac{1}{N-1} \operatorname{Tr}\left[\left(\hat{\rho}_{1}^{2} / 2-\hat{\rho}_{1}\right) \hat{I}\right] .
$$

The percentage of current carried by the correlation terms is indicated in Fig. 3 by the filled symbols. In line with our previous observations, it is characterized by a single value for each oligomer. The correlation contribution remains a small fraction of the total current as a function of applied voltage. These findings imply the existence of an approximate single-determinantal current-carrying wave function that captures most of the contributions to electron flow even when the HF approximation begins to fail.

The HF approximation determines the best orbitals for a $\left|\Psi_{1-\text { det }}\right\rangle$ that minimizes the energy. Since the current largely reflects the form of the wave function, it is plausible that an approximate state that maximizes overlap with the currentcarrying wave function may improve the orbital description of an independent-particle model. Truncated configuration expansions built from the eigenfunctions of the one-body density matrix, the natural orbitals (NOs), are known to maximize overlap with $\left|\Psi_{M B}\right\rangle$. Since there are only $N / 2$ NOs with large occupation numbers $n_{i}$-in agreement with the calculated small correlation measure $C$ in our MMJs-we use the single determinant made by doubly occupying the orbitals with occupation numbers close to 2 ; note that an exact treatment of maximizing the overlap for $\left|\Psi_{1 \text {-det }}\right\rangle$ yields the Brueckner determinant. We find that the current carried, $I_{\mathrm{NO}}$, is close to $I_{\mathrm{MB}}$ as shown in Fig. 3 where the percentage of the remaining differences is plotted versus molecular length. Remarkably, these deviations are of the same order as the correlation contributions determined by Eq. (5). Indeed, in Fig. 4 the current-voltage characteristics clearly demonstrate that the "maximum overlap model" yields almost indistinguishable results from the many-body calculations independent of residual electron correlations; the HF singleelectron picture already shows significant deviation for pentane [Fig. 4(a)], which grows much larger for the silanes [Fig. 4(b)]. The use of the natural orbitals in defining $\left|\Psi_{1 \text {-det }}\right\rangle$ acquires a practical significance in view of recent advances allowing their construction from one-electron equations with an effective potential. ${ }^{19}$

In summary, we have shown within a many-body scheme how an independent-particle description emerges for electrons tunneling across the barrier of insulating materials, due to zero-bias correlations being such that a single-determinant wave function is appropriate. Our main conclusion is that a strong selection criterion for a single particle transport model is to maximize the overlap with the many-body state; this does not yield the Hartree-Fock determinant. Indeed, this Slater determinant outperforms the HF one substantially. As most KS DFT implementations to date have recently been shown to be performed at essentially the Hartree level, ${ }^{1}$ the independent-particle picture we have established here is better than both present models and, hence, is valid beyond the limits of conventional electronic structure methods for quantum transport.

\section{ACKNOWLEDGMENTS}

We would like to thank T. M. Henderson for many helpful discussions, and assistance by A. Uskova and S. D. Elliott is acknowledged. This work was funded by Science Foundation Ireland.
${ }^{1}$ M. Koentopp, K. Burke, and F. Evers, Phys. Rev. B 73, 121403(R) (2006).

${ }^{2}$ F. Evers, F. Weigend, and M. Koentopp, Phys. Rev. B 69, 235411 (2004)

${ }^{3}$ C. Toher, A. Filippetti, S. Sanvito, and K. Burke, Phys. Rev. Lett. 95, 146402 (2005).

${ }^{4}$ Introducing Molecular Electronics, edited by G. Cuniberti, G. Fagas, and K. Richter (Springer, Berlin, 2005).

${ }^{5}$ P. Delaney and J. C. Greer, Phys. Rev. Lett. 93, 036805 (2004); P. Delaney and J. C. Greer, Int. J. Quantum Chem. 100, 1163 (2004).

${ }^{6}$ Z. Zhang, C. F. Destefani, C. McDonald, and T. Brabec, Phys. Rev. B 72, 161309(R) (2005).

${ }^{7}$ M. Albrecht, B. Song, and A. Schnurpfeil, cond-mat/0512554 (unpublished).

${ }^{8}$ Y. Xue, S. Datta, and M. A. Ratner, Chem. Phys. 281, 151 (2002).

${ }^{9}$ G. Fagas, A. Kambili, and M. Elstner, Chem. Phys. Lett. 389, 268 (2004)
${ }^{10}$ S. Kümmel, L. Kronik, and J. P. Perdew, Phys. Rev. Lett. 93, 213002 (2004).

${ }^{11}$ N. Sai, M. Zwolak, G. Vignale, and M. Di Ventra, Phys. Rev. Lett. 94, 186810 (2005).

${ }^{12}$ J. Bardeen, Phys. Rev. Lett. 6, 57 (1961).

${ }^{13}$ F. Zahid, M. Paulsson, E. Polizzi, A. W. Ghosh, L. Siddiqui, and S. Datta, J. Chem. Phys. 123, 064707 (2005).

${ }^{14}$ J. C. Greer, J. Comput. Phys. 146, 181 (1998).

${ }^{15}$ Convergence of the results has been checked against the increase of the configuration-interaction expansion. For the reported calculations, the number of CSFs is around $10^{4}$.

${ }^{16}$ B. Q. Xu and N. J. Tao, Science 301, 1221 (2003); X. Y. Xiao, B. Q. Xu, and J. Tao, Nano Lett. 4, 267 (2004).

${ }^{17}$ A. Salomon, D. Cahen, S. Lindsay, J. Tomfohr, V. B. Engelkes, and C. D. Frisbie, Adv. Mater. (Weinheim, Ger.) 15, 1881 (2003).

${ }^{18}$ W. Haiss. R. J. Nichols, H. van Zalinge, S. J. Higgins, D. Bethell, and D. J. Schiffrin, Phys. Chem. Chem. Phys. 6, 4330 (2004).

${ }^{19}$ K. Pernal, Phys. Rev. Lett. 94, 233002 (2005). 